at baseline in responders to Droglican according to the OMERACT-OARSI criteria compared to non-responders $(76,11 \pm 53,25$ vs $104,25 \pm 84,93 ; n=171$ vs 46; $p=0,047$ ), meanwhile the values for APOA2 appeared statistically increased in responders with a $50 \%$ reduction in WOMAC pain score compared to nonresponders $(79,95 \pm 58,53$ vs $66,05 \pm 46,49 ; \mathrm{n}=129$ vs $112 ; p=0,028)$. Patients with lower levels of ORM2 (median concentration $=69,8 \mathrm{ug} / \mathrm{mL}$ ) and/or higher level of APOA2 (median concentration $=63,8 \mathrm{ug} / \mathrm{mL}$ ) showed a markedly better response to pharmacotherapy. Statistical interactions between ORM2 and APOA2 levels and radiologic $\mathrm{K} / \mathrm{L}$ grade were also detected ( $p=0,048$ and $p=0,002$ respectively). No statistically significant differences were found for the other four proteins.

Conclusions: Our results show that ORM2 and APOA2 levels significantly correlates with patients response to Droglican suggesting the possibility of their use in predictive assays in order to optimize therapeutic outcomes in OA. Validation studies in different cohorts are needed to identify and validate a cut-off point for these biomarkers.

Disclosure of Interest: V. Calamia: None declared, M. Camacho-Encina: None declared, L. González-Rodríguez: None declared, P. Fernández-Puente: None declared, I. Rego-Pérez: None declared, M. Herrero Employee of: Bioiberica S.A., H. Martínez Employee of: Bioiberica S.A., C. Ruiz-Romero: None declared, F. J. Blanco: None declared

DOI: 10.1136/annrheumdis-2017-eular.6224

\section{AB1175 DECREASED AUTOPHAGIC ACTIVITY IN T LYMPHOCYTES FROM PATIENTS WITH NEWLY DIAGNOSED SYSTEMIC LUPUS ERYTHEMATOSUS}

$\underline{\text { X. Luo }}^{1}$, Y. Liu ${ }^{2}$, M. Yang ${ }^{2} .{ }^{1}$ Department of Rheumatology; ${ }^{2}$ West China Hospital, Sichuan University, Chengdu, China

Background: Alterations in T-lymphocyte homeostasis have been suggested to play a key role in the pathogenesis of SLE. Autophagy is now emerging as a core player in the development and the functioning of the immune system.

Objectives: we investigated the autophagic behavior of $T$ cells from patients with SLE.

Methods: Thirty patients with SLE and twenty-five healthy subjects matched for gender and age were recruited. The levels of mRNA encoding ATG5, ATG7, Beclin-1 and LC3 was determined by quantitative real-time polymerase chain reaction (qPCR), and evaluate autophagy activity in T cells by flow cytometry. The number of autophagic structures was examined by TEM in T cells from SLE patients and healthy controls.

Results: We documented a decreased of mRNA expression of LC3 and Atg7 in T cells from patients with SLE ( $t=2.282, P=0.027 ; t=3.573, P=0.001)$.A decreased percentage of autophagic cells was comfirmed in $T$ cells from patients with SLE, as compared to healthy donors by flow cytometry $(t=2.034, P=0.047$ ).no significant correlations between autophagy levels in $\mathrm{T}$ cells and the disease activity of patients were observed $(p>0.05)$.

Conclusions: Our results indicate that autophagy activity in T cells from SLE patients is decreased, which may contribute to the development of SLE, and thus that resetting autophagic activity may be an important therapeutic goal in this autoimmune disease.

Acknowledgements: The authors thank Dr Liu Qinsong, and Mr Xia Yanhui for their technical assistance.

Disclosure of Interest: None declared

DOI: 10.1136/annrheumdis-2017-eular.2558

\section{AB1176 ANTI-MX1 ANTIBODY: A NEW POTENTIAL BIOMARKER EXPANDING THE CONCEPT OF INTERSTITIAL PNEUMONIA WITH AUTOIMMUNE FEATURES (IPAF)}

Y. Hamano ${ }^{1}$, H. Kida ${ }^{1}$, A. Murakami ${ }^{2}$, M. Yanagawa ${ }^{3}$, K. Ueda $^{3}$, O. Honda ${ }^{3}$, Y. Kato ${ }^{1}$, H. Takamatsu ${ }^{1}$, N. Tomiyama ${ }^{3}$, A. Kumanogoh ${ }^{1}$. ${ }^{1}$ Department of Respiratory Medicine, Allergy and Rheumatic Diseases, Osaka University, Suita; ${ }^{2}$ Medical \& Biological Laboratories Co., Ltd., Ina Laboratory, Ina; ${ }^{3}$ Department of Radiology, Osaka University, Suita, Japan

Background: "Interstitial pneumonia with autoimmune features (IPAF)" is the updated concept instead of UCTD-ILD or lung-dominant CTD, classifying idiopathic interstitial pneumonias (IIPs) with underlying autoimmune processes by the presence of a combination of features from three domains; clinical, serologic, and morphologic domains. In IIPs, idiopathic non-specific interstitial pneumonia (INSIP) and idiopathic pulmonary fibrosis are often difficult to distinguish without surgical lung biopsy. We discovered autoantibody against myxovirus resistance protein-1 (MX1), type I interferon-inducible protein, as the biomarker specific for INSIP and associated with favorable prognosis [1]. We also reported that some of patients with collagen vascular diseases (CVDs) possess anti-MX1 antibody only when complicated with interstitial lung disease [2]

Objectives: This study was aimed to investigate the association of anti-MX1 antibody positivity with IPAF category in patients with IIPs and their clinical characteristics through a cross-sectional study. We also assessed the potential of anti-MX1 antibody to expand the concept of autoimmunity in IIPs.

Methods: Anti-MX1 antibodies in sera of consecutive Japanese patients with chronic fibrosing IIPs $(n=114)$, who visited the outpatient office of Osaka University Hospital from February to October 2014, were measured using ELISA.
IPAF patients were classified according to the IPAF criteria in 2015 ERS/ATS statement. Comparison of the patients' clinical characteristics and high-resolution computed tomography (HRCT) findings evaluated by three thoracic radiologists blinded to the clinical information were statistically analyzed. Serum IFN $\alpha$ was measured using ELISA.

Results: Among 114 patients with IIPs, 20 patients (17.5\%) were positive for anti-MX1 antibody and 33 patients $(28.9 \%)$ were classified as IPAF. When IPAF patients $(n=33)$ were compared with non-IPAF patients $(n=81)$, IPAF was associated with female, higher level of C-reactive protein (CRP), the presence of HRCT findings of "predominantly peribronchovascular distribution" and the absence of two HRCT findings of "honeycombing' and 'traction bronchiectasis". Of 81 non-IPAF patients, 13 patients $(16.0 \%)$ were anti-MX1 antibody positive. Among them, if anti-MX1 autoantibody were included into the existent serological domain in the IPAF criteria, 8 patients $(9.9 \%)$ would be classified as IPAF. Anti-MX1 antibody positivity did not correlate with IPAF category. In non-IPAF patients, antiMX1 antibody-positive patients were associated with female and predominantly smaller 'spared area' when compared to anti-MX1 antibody-negative patients. Serum IFN $\alpha$ concentration was not associated with either anti-MX1 antibody positivity or IPAF category.

Conclusions: A substantial number of patients classified as non-IPAF were positive for anti-MX1 antibody, suggesting that this new autoantibody could have the potential expanding the definition of IPAF. The further studies for the clinical course and drug efficacy of anti-MX1 antibody-positive non-IPAF patients must be explored.

References:

[1] Hamano $Y$ et al. Classification of idiopathic interstitial pneumonias using anti-myxovirus resistance-protein 1 autoantibody. Scientific Reports 2017 (in press).

[2] Ann Rheum Dis 2014;73(Suppl2): 1139

Disclosure of Interest: Y. Hamano: None declared, H. Kida: None declared, A. Murakami Employee of: Medical \& Biological Laboratories Co., Ltd., Ina Laboratory, M. Yanagawa: None declared, K. Ueda: None declared, O. Honda: None declared, Y. Kato: None declared, H. Takamatsu: None declared, N. Tomiyama: None declared, A. Kumanogoh: None declared

DOI: 10.1136/annrheumdis-2017-eular.4280

\section{Rehabilitation}

\section{AB1177 THE COMBINATION OF PHYSIOTHERAPY AND BIOLOGICAL THERAPY FOR THE MANAGEMENT OF ANKYLOSING SPONDYLITIS}

A. Sehimi, Y. Slimani, N. Messaoud, O. Bensaber. Physical Medicine and Rehabilitation Department, University Hospital and Medicine Faculty of Sidi Bel Abbes, Sidi Bel Abbes, Algeria

Background: The management of the ankylosing spondylitis (AS) aims at relieving patients'pain, restoring their joint mobility and preventing structural damage which results in progressive deformity, in order to improve the functional status and quality of life of these patients, using various pharmacological and non-pharmacological means. The importance of the physiotherapy in patients with AS under biological treatment was reported in some studies, but the literature on this topic is still scarce.

Objectives: Report the experience, of our department of Physical and Rehabilitation Medicine, in the management of the AS, especially the effect of the combination of physiotherapy and biological therapy on pain, disease activity, spinal mobility, functional capacity and quality of life.

Methods: Prospective study on 20 patients diagnosed with AS, treated with tumor necrosis factor alpha inhibitors (TNFa inhibitors) and placed under physiotherapy for 3 months. At baseline and at the end of 3 months, we evaluated Bath AS Disease Activity Index (BASDAI), occiput-wall distance, Hirtz index, Schober index, Bath AS Functional Index (BASFI) and Visual Analog Scale (VAS) of patient's quality of life.

Results: The 20 patients (9 females), aged 38.4 years \pm 10.24 [range 19-55], treated with TNFa inhibitors (Etanercept in $35 \%$ and Adalimumab in 65\%) and included in a physiotherapy program of 3 months (3 sessions/week), comprising muscle relaxation, flexibility exercises for cervical, thoracic and lumbar spine, range of motion exercises of coxofemoral joints, muscular strengthening, straight posture and respiratory exercises.

After 3 months, all outcome parameters showed statistically significant improvements $(P<0,05)$, as shown in the folliwing table.

\begin{tabular}{lccc}
\hline & & \multicolumn{2}{c}{ Paired Diffrences } \\
\cline { 3 - 4 } & & Mean & $P$ value \\
\hline Pair 1 & BASDAlbaseline - BASDAImonth3 & 3,44615 & 0,000 \\
Pair 2 & BASFlbaseline - BASFImonth3 & 2,9654 & 0,000 \\
Pair 3 & OWIbaseline - OWImonth3 & 1,7125 & 0,023 \\
Pair 4 & SCHOBERbaseline - SCHOBERmonth3 & $-1,5667$ & 0,000 \\
Pair 5 & HIRTZbaseline - HIRTZmonth3 & $-0,7273$ & 0,001 \\
Pair 6 & VASpatient_baseline - VASpatient_month3 & 4,0833 & 0,000 \\
\hline
\end{tabular}

Conclusions: According to our results, the combination of physiotherapy and 
biological therapy is an effective mean in increasing functional capacity and joint mobility, decreasing disease activity, improving quality of life for AS patients. Currently available data do not adequately address what role physiotherapy may have on patients with AS receiving biological drugs [1]. There are a few studies evaluating the effects of exercises in patients with AS receiving TNFa inhibitors [2,3]. References:

[1] Elyan M, Khan MA (2008) Does physical therapy still have a place in the treatment of ankylosing spondylitis? Curr Opin Rheumatol 20:282-286.

[2] Dubey SG, Leeder J, Gaffney K (2008) Physical therapy in anti-TNF treated patients with ankylosing spondylitis. Rheumatology (Oxford) 47:1100-1101.

[3] Lubrano E, D'Angelo S, Parsons WJ, Serino F, Tanzillo AT,Olivieri I, Pappone N (2006) Effects of a combination treatment of an intensive rehabilitation program and etanercept in patients with ankylosing spondylitis: a pilot study. $\mathrm{J}$ Rheumatol 33:2029-2034.

Disclosure of Interest: None declared

DOI: 10.1136/annrheumdis-2017-eular.5453

\section{AB1178 A PROSPECTIVE STUDY ON THE EFFECTS OF 3-MONTH COURSES OF TRADITIONAL PHYSIOTERAPY AND YOGA IN PATIENTS WITH CHRONIC ARTHRITIS AND PRIMARY FIBROMYALGIA}

C. Nalli ${ }^{1}$, L. Andreoli ${ }^{1}$, R. Avanzini ${ }^{2}$, P. Tipa ${ }^{2}$, M. Zanetti ${ }^{2}$, C. Milini ${ }^{3}$, S. Olivieri ${ }^{4}$, E. Abrami ${ }^{5}$, R. Furfari ${ }^{5}$, R. Gorla ${ }^{1}$, A. Tincani ${ }^{1} .{ }^{1}$ Rheumatology and Clinical Immunology Unit, Spedali Civili and University of Brescia; ${ }^{2}$ Physical Therapy Student, University of Brescia; ${ }^{3}$ Rehabilitation Unit, Spedali Civili; ${ }^{4}$ Accademia Essse: ${ }^{5}$ Associazione Bresciana Artrite Reumatoide (ABAR) Onlus, Brescia, Italy

Background: Physical activity is of fundamental importance for people with rheumatic diseases (RD). The traditional approach of Physiotherapy (PT) has been placed side by side with other physical programs, such as Yoga $(Y)$, aimed at reducing chronic pain and improving the quality of life.

Objectives: To evaluate the effects of PT and $Y$ on patients with inflammatory and non-inflammatory RD: Chronic Arthritis (CA) and Primary Fibromyalgia (FM). Methods: Patients were enrolled in a prospective study including 3 months of PT and 3 months of $Y$ (with a specific focus on the control of breathing Ai-jutsu). Each activity was performed bi-weekly in a dedicated facility. Patients were randomly allocated to either the group starting with PT or that starting with Y. After 3 months they switched to the other activity. At the beginning and at the end of each activity patients underwent a medical assessment of their physical status and were proposed questionnaires: 1) HAQ for disability in everyday life; 2) ZUNG self-rating depression scale; 3) Tampa scale for Kinesiophobia assessing the fear and avoidance of movement. Patients with FM compiled also the FIQ for the impact of FM in everyday life. All patients rated their physical pain by VAS (Visual Analogic Scale) from 0 to 10.

Results: Thirteen patients with CA ( $77 \%$ female, median age 62 years, median disease duration 19 years) and 8 with FM (100\% female, 56 years, 9 years) participated in the study. At baseline, there were no differences between CA and FM patients in terms of ZUNG, HAQ and Tampa scores. After 3 and 6 months of activity, all items had a tendency toward improvement, with a statistically significant reduction for VAS in both groups (nearly -30\%). By intra-group comparison between the beginning and the end of each activity, we observed that patients with CA had a significant reduction of ZUNG and HAQ scores (50 vs 40.5 and 0.600 vs 0.475 , respectively) during PT activity, while FM patients had a trend toward the increase of Tampa score (24.5 vs 34.5). During $Y$ activity, significant reductions were observed in VAS score for CA patients (5 vs 2.5) and in Tampa score for FM patients (34.5 vs 23.5). Overall, all items had a tendency toward improvement for FM patients during $\mathrm{Y}$ activity.

Conclusions: This pilot study involving both patients with inflammatory and noninflammatory RD demonstrated benefit from an integrated program of sequential PT and $Y$ and highlighted differences in patients' needs according to their disease type. Particularly, PT seemed to bring more benefit to CA patients, probably because of the individualized work on joint movement range and muscular strengthening, while FM patients may have had a negative impact by this approach, as seen by the increase in the fear of movement. Conversely, FM patients had a tendency toward improvement during $Y$ activity.

Acknowledgements: We thank the Patients Association (ABAR) for promoting and supporting the activities of this study and Essse Accademia for gently introducing our patients to the world of Yoga.

Disclosure of Interest: C. Nalli: None declared, L. Andreoli: None declared, R. Avanzini: None declared, P. Tipa: None declared, M. Zanetti: None declared, C. Milini: None declared, S. Olivieri Employee of: Accademia Essse, E. Abrami: None declared, R. Furfari: None declared, R. Gorla: None declared, A. Tincani: None declared

DOI: 10.1136/annrheumdis-2017-eular.4376

\section{AB1179 CAN THE PATIENTS WITH TOTAL KNEE ARTHROPLASTY ACHIEVE DISCHARGE CRITERIA FOR KNEE FLEXION}

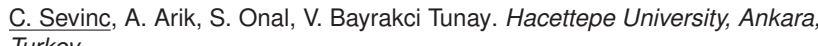
Turkey

Background: Knee range of motion (ROM) at discharge following total knee arthroplasty is used as a clinical indicator of performance. At least 90 degrees of flexion is standard discharge criteria for many hospitals.

Objectives: To investigate whether the discharge criteria is realistic in patients with TKA.

Methods: 13 patients ( $11=$ female, $2=$ male) who underwent unilateral TKA were included in this study. Mean age was $68.46 \pm 7.52$ years, mean body mass index (BMI) was $30.14 \pm 5.6 \mathrm{~kg} / \mathrm{m}^{2}$. Hospitalization duration was recorded. Knee flexion and extension degree were measured actively with goniometer during heel sliding on discharge day. Oxford Knee Score (OKS) was used to evaluate physical function and pain before surgery. Overall scores ranges from 0 (worst) to 48 (best). Descriptive statistics were used to analyse the data using SPSS v22.

Results: Hospitalization duration was $5.07 \pm 1.25$ days. Knee flexion degree and knee extension limitation were found $79.15 \pm 20.84^{\circ}$ and $12.92 \pm 7.31^{\circ}$, respectively. Oxford Knee Score was calculated $19 \pm 8.30$ point. Patients' results were given at Table 1.

Table 1. Patients' results

\begin{tabular}{lccc}
\hline Patients & Knee flexion degree $\left(^{\circ}\right)$ & Extension limitation $\left(^{\circ}\right)$ & Oxford Knee Score \\
\hline 1 & 65 & 11 & 28 \\
2 & 98 & 18 & 33 \\
3 & 94 & 12 & 15 \\
4 & 88 & 10 & 17 \\
5 & 100 & 24 & 22 \\
6 & 36 & 24 & 14 \\
7 & 86 & 12 & 15 \\
8 & 70 & 15 & 17 \\
9 & 90 & 2 & 12 \\
10 & 80 & 10 & 36 \\
11 & 42 & 10 & 12 \\
12 & 80 & 0 & 10 \\
13 & 100 & &
\end{tabular}

Conclusions: Patients have noticeable extension limitations at discharge. It should follow in the upcoming days. Nearly half of the patients flexed their knee less than expected. Due to the fact that discharge criteria for knee flexion is unrealistic. It should be investigate whether patients with less knee flexion degree can gain function as patients with more knee flexion degree.

\section{References:}

[1] Husted, Henrik, et al. Traditions and myths in hip and knee arthroplasty: A narrative review. Acta orthopaedica 85.6 (2014): 548-555.

[2] Naylor, Justine M., et al. Is discharge knee range of motion a useful and relevant clinical indicator after total knee replacement? Part 1. Journal of evaluation in clinical practice 18.3 (2012): 644-651.

Disclosure of Interest: None declared

DOI: 10.1136/annrheumdis-2017-eular.6397

\section{AB1180 THE INTENSIVE EXERCISE PROGRAMME FOR NON-RADIOGRAPHIC AXIAL SPONDYLOARTHRITIS AND FOR ANKYLOSING SPONDYLITIS MAY IMPROVED QUALITY OF LIFE AND DISEASE ACTIVITY}

M. Husakova ${ }^{1}$, K. Pavelka ${ }^{1}$, M. Spiritovic ${ }^{1,2}$, A. Levitova ${ }^{1,2}{ }^{1}{ }^{1}$ nstitute of Rheumatology and Department of Rheumatology, First Faculty of Medicine, Charles University, Prague 2; ${ }^{2}$ Charles University, Faculty of Physical Education and Sport, Prague, Czech Republic

Background: The therapy for axial spondyloarthritis (axSpA) is complex. Although anti-inflammatory medication is necessary for axSpA treament, the exercise therapy is required to maintain mobility. The limited data are available to evaluate the effect of exercise therapy on quality of life in axSpA, particularly in patients with the non-radiographic form of the disease (nr-axSpA).

Objectives: To investigated the quality of life in axSpA subgroups, nr-axSpA and Ankylosing spondylitis (AS) in response to intensive rehabilitation programme Methods: 46 patients with axSpA characterised according to criteria of Assessment of SpondyloArthritis international Society (ASAS) as nr-axSpA $(n=23)$ and AS $(n=23)$ with stable disease and treatment underwent 24 weeks long intervention. The intervention consisted in twice a week outpatient group physiotherapy as exercise units of 60 minutes and a daily home-based exercise programme. All outcomes, disease activity (Bath AS Disease Activity Index, BASDAI and AS Disease Activity Index, ASDAS-CRP) and quality of life (AS quality of life, ASQoL and European quality of life, EurQoL) as well as patients self-reported outcomes such as "patients global assessment" and "pain assessment" were measured at baseline and at the end of exercise program.

Results: Altogether, 41 axSpA patients (AS, $n=22$ and $n r-a x S p A, n=19$ ) finished complete six months programme. The disease activity was improved in all axSpA patients (ASDAS-CRP $2.08 \pm 0.12$ to $1.83 \pm 0.11, p<0.01$ ), particularly in $\mathrm{nr}$-axSpA subgroup, ASDAS-CRP $(1.98 \pm 0.19$ to $1.71 \pm 0.15, \mathrm{p}<0.05)$. There were no differences in the changes in ASDAS-CRP and BASDAl over the exercise training between groups (data not shown). After exercise therapy, positive changes of "Patients global assessment", were evaluated by patients of both subgroups, nr-ax-SpA $(33.42 \pm 5.13$ to $23.68 \pm 4.11, p<0.01)$ and AS $(35.22 \pm 3.94$ to $25.2 \pm 2.92$, $\mathrm{p}<0.01)$. The "assessment of pain during the last 7 days", however, was improved only by patients in the nr-axSpA subgroup $(34.74 \pm 5.88$ vs. $21.05 \pm 4.71, p<0.05)$. The quality of life, ASQoL was not changed after rehabilitation programme. 